\title{
Metal abundances in the hottest known DO white dwarf (KPD 0005+5106) ^
}

\author{
D. Wassermann ${ }^{1}$, K. Werner ${ }^{1}$, T. Rauch ${ }^{1}$, and J. W. Kruk ${ }^{2, \star \star}$ \\ ${ }^{1}$ Institute for Astronomy and Astrophysics, Kepler Center for Astro and Particle Physics, Eberhard Karls Universität Tübingen, \\ Sand 1, 72076 Tübingen, Germany \\ e-mail: werner@astro.uni-tuebingen.de \\ 2 Department of Physics and Astronomy, Johns Hopkins University, Baltimore, MD 21218, USA
}

Received 17 May 2010 / Accepted 24 July 2010

\begin{abstract}
We present the analysis of ultraviolet and optical spectra of KPD $0005+5106$. Redetermination of the atmospheric parameters is necessary because the recent discovery of highly ionised metals (Ne VIII, CaX) indicates that the effective temperature is significantly higher than previously thought. Here we announce the discovery of lines from highly ionised silicon, sulphur, and iron (Si VII, S VII, $\mathrm{Fe} \mathrm{X}$ ) which were never found before in any stellar photosphere. Our analysis of these lines and those from helium and several other metals gives $T_{\text {eff }}=200000 \pm 20000 \mathrm{~K}, \log g=6.7 \pm 0.3$. Mass and luminosity follow from a comparison with evolutionary tracks: $M=0.64 M_{\odot}$ and $\log L / L_{\odot}=3.7$, so that, strictly speaking, the star is a helium-burning pre-white dwarf. The mass fractions of the metals in the helium-dominated atmosphere are in the range $0.7-4.3$ solar. Hydrogen is not detectable and we derive an upper abundance limit of 0.034 solar. This abundance pattern is probably unaffected by gravitational settling and radiative levitation. Its origin lies in previous evolutionary stages. We discuss the link of KPD $0005+5106$ to RCrB stars and the possibility that it results from a double-degenerate merger event.
\end{abstract}

Key words. stars: abundances - stars: atmospheres - stars: evolution - stars: AGB and post-AGB - white dwarfs stars: individual: KPD 0005+5106

\section{Introduction}

Since its discovery (Downes et al. 1985) the He-rich DO white dwarf (WD) KPD 0005+5106 has attracted particular attention because, unlike any other WD, it displays sharp emission lines. A first comprehensive NLTE model-atmosphere analysis (Werner et al. 1994) showed that KPD 0005+5106 is the hottest known DO $\left(T_{\text {eff }}=120000 \mathrm{~K}\right)$. In subsequent years, many new DOs were discovered, in particular by the Sloan Digital Sky Survey, and some of them challenged the role of KPD $0005+5106$ as the hottest representative of its class (Hügelmeyer etal. 2006). High-resolution spectroscopy with the Far Ultraviolet Spectroscopic Explorer (FUSE), however, pointed at an even higher $T_{\text {eff }}$ for KPD $0005+5106$. The discovery of Ne VIII and Ca X lines suggested $T_{\text {eff }} \approx 200000 \mathrm{~K}$ and preliminary modelling showed that this high $T_{\text {eff }}$ also improves the model fit to the He II line spectrum (Werner et al. 2007, 2008). This solved a problem connected with a number of emission features that were previously identified as $\mathrm{O}$ VIII lines. These would require extremely high temperatures $\left(\approx 10^{6} \mathrm{~K}\right)$ so that their origin was attributed to shock fronts in a stellar wind. Instead, it turned out that these features are Ne VIII lines that form in the hot photosphere.

* Based on observations made with the NASA-CNES-CSA Far Ultraviolet Spectroscopic Explorer. FUSE was operated for NASA by the Johns Hopkins University under NASA contract NAS5-32985.

$\star \star$ Current address: NASA Goddard Space Flight Center, Greenbelt, MD 20771, USA.
We perform a systematic analysis of UV and optical spectra to constrain $T_{\text {eff }}, \log g$, and element abundances. Determination of the element abundance pattern offers the possibility of connecting KPD $0005+5106$ to other He-rich post-AGB stars in order to establish the possible existence of another He-rich evolutionary sequence in parallel to the [WC]-PG1159 phases. Going beyond our exploratory work (Werner et al. 2007), we use here NLTE model atmospheres with extended model atoms for all identified species. We perform abundance determinations for metals (C, N, O, Ne, Si, S, Ca, Fe) and derive an upper limit for H. In our previous study (Werner et al. 1994) we examined $\mathrm{C}, \mathrm{N}, \mathrm{O}$, but the results became spurious with the revision of $T_{\text {eff }}$. As before, our analysis utilises Hubble Space Telescope (HST) GHRS and FOS UV spectra and medium-resolution optical spectroscopy. In addition we now have far-UV FUSE spectra available plus high-resolution optical spectra obtained with the $10 \mathrm{~m}$ Keck telescope. FUSE spectroscopy turned out to be the most useful observation.

In the next part (Sect. 2) we present an overview of the observational data and perform a detailed line identification. This is worthwhile because KPD $0005+5106$ has the most highly ionised photosphere of any star known (aside from neutron stars, of course), and thus serves as a reference for any future discovery of similar objects. In particular, we have found for the first time lines of Si VII, S VII, and Fe X, whose mere presence confirms the high $T_{\text {eff }}$.

We then describe our model atmospheres in some detail with respect to the design of our NLTE model atoms (Sect. 3). The 
Table 1. Ultraviolet photospheric lines $(\lambda<3000 \AA$, vacuum wavelengths) identified in KPD 0005+5106.

\begin{tabular}{|c|c|c|c|c|c|c|}
\hline \multicolumn{3}{|c|}{ Wavelength / $\AA$} & \multirow{2}{*}{$\begin{array}{l}\text { Ion } \\
\text { Si VII }\end{array}$} & \multicolumn{3}{|c|}{ Transition } \\
\hline 923.4 & & & & $3 \mathrm{p}^{\prime 3} \mathrm{D}_{3}$ & - & $3 d^{\prime 3} F_{3}^{o}$ \\
\hline 944.52 & & & S VI & $3 \mathrm{~s}^{2} \mathrm{~S}_{1 / 2}$ & - & $3 p^{2} \mathrm{P}_{1 / 2}^{\mathrm{o}^{3}}$ \\
\hline 958.70 & & & He II & 2 & - & 9 \\
\hline 972.11 & & & He II & 2 & - & 8 \\
\hline 979.3 & & & $\mathrm{Fe} X$ & $4 \mathrm{~s}^{2} \mathrm{D}_{5 / 2}$ & - & $4 p^{2} F_{7 / 2}^{O}$ \\
\hline 992.36 & & & He II & 2 & - & 7 \\
\hline 1025.27 & & & He II & 2 & - & 6 \\
\hline 1031.91 & & & O VI & $2 \mathrm{~s}^{2} \mathrm{~S}_{1 / 2}$ & - & $2 \mathrm{p}^{2} \mathrm{P}_{3 / 2}^{\mathrm{o}}$ \\
\hline 1005.7 & & & Si VII & $3 \mathrm{~s}^{\prime \prime}{ }^{3} \mathrm{P}_{2}^{\mathrm{o}}$ & - & $3 \mathrm{p}^{\prime \prime}{ }^{3} \mathrm{P}_{1}$ \\
\hline 1022.9 & & & Fex & $4 s^{2} D_{3 / 2}$ & - & $4 p^{2} F_{5 / 2}^{o}$ \\
\hline 1051.73 & & & S VII & $3 \mathrm{~s}^{3} \mathrm{P}_{2}^{\mathrm{o}}$ & - & $3 p^{3} S_{1}$ \\
\hline 1052.3 & & & Si VII & $3 p^{\prime}{ }^{3} P_{2}$ & - & $3 \mathrm{~d}^{\prime}{ }^{3} \mathrm{P}_{2}^{\mathrm{o}}$ \\
\hline 1060.36 & - & 1061.03 & Ne VIII & $5 s^{2} S$ & - & $6 \mathrm{p}^{2} \mathrm{P}^{\mathrm{o}}$ \\
\hline 1084.94 & & & He II & 2 & - & 5 \\
\hline 1107.59 & - & 1107.98 & C IV & $3 \mathrm{p}^{2} \mathrm{P}^{\mathrm{o}}$ & - & $4 d^{2} D$ \\
\hline 1117.76 & & & S VI & $4 \mathrm{f}^{2} \mathrm{~F}^{\mathrm{O}}$ & - & $5 g^{2} G$ \\
\hline 1122.33 & - & 1122.61 & O VI & $4 d^{2} D$ & - & $5 \mathrm{f}^{2} \mathrm{~F}^{\mathrm{o}}$ \\
\hline 1124.70 & - & 1124.82 & OVI & $4 \mathrm{f}^{2} \mathrm{~F}^{\mathrm{o}}$ & - & $5 g^{2} G$ \\
\hline 1129.02 & - & 1130.35 & Ne VIII & $5 \mathrm{p}^{2} \mathrm{P}^{\mathrm{o}}$ & - & $6 d^{2} D$ \\
\hline 1132.8 & & & Si VII & $3 s^{\prime}{ }^{3} \mathrm{D}_{3}^{\mathrm{o}}$ & - & $3 p^{\prime}{ }^{3} F_{4}$ \\
\hline 1135.4 & & & Si VII & $3 s^{5} S_{2}^{o}$ & - & $3 \mathrm{p}^{5} \mathrm{P}_{3}$ \\
\hline 1136.60 & & & $\mathrm{CaX}$ & $4 p^{2} \mathrm{P}_{1 / 2}^{\mathrm{o}}$ & - & $4 d^{2} D_{3 / 2}$ \\
\hline 1137.2 & & & Si VII & $3 s^{\prime}{ }^{3} \mathrm{D}_{2}^{\mathrm{o}}$ & - & $3 \mathrm{p}^{\prime}{ }^{3} \mathrm{~F}_{3}$ \\
\hline 1142.4 & & & Si VII & $3 s^{5} S_{2}^{o}$ & - & $3 \mathrm{p}^{5} \mathrm{P}_{2}$ \\
\hline 1146.5 & & & Si VII & $3 s^{5} S_{2}^{o}$ & - & $3 p^{5} \mathrm{P}_{1}$ \\
\hline 1155.8 & & & Si VII & $3 \mathrm{p}^{\prime}{ }^{3} \mathrm{P}_{1}$ & - & $3 \mathrm{~d}^{\prime}{ }^{3} \mathrm{D}_{2}^{\mathrm{o}}$ \\
\hline 1156.0 & & & Si VII & $3 \mathrm{p}^{\prime 1} \mathrm{D}$ & - & $3 \mathrm{~d}^{\prime 1} \mathrm{D}^{\mathrm{o}}$ \\
\hline 1158.17 & & & Si VII & $3 p^{\prime \prime}{ }^{1} D$ & - & $3 \mathrm{~d}^{\prime \prime}{ }^{1} \mathrm{D}^{\mathrm{o}}$ \\
\hline 1159.30 & & & $\mathrm{CaX}$ & $4 \mathrm{p}^{2} \mathrm{P}_{3 / 2}^{\mathrm{o}}$ & - & $4 d^{2} D_{5 / 2}$ \\
\hline 1162.24 & - & 1162.67 & Ne VIII & $5 d^{2} D$ & - & $6 \mathrm{f}^{2} \mathrm{~F}^{\mathrm{o}}$ \\
\hline 1164.54 & - & 1164.75 & Ne VIII & $5 \mathrm{f}^{2} \mathrm{~F}^{\mathrm{o}}$ & - & $6 g^{2} G$ \\
\hline 1164.88 & & & $\mathrm{Ne}$ VIII & $5 g^{2} G$ & - & $6 \mathrm{~h}^{2} \mathrm{H}^{\mathrm{o}}$ \\
\hline 1165.94 & - & 1166.18 & Ne VIII & $5 \mathrm{f}^{2} \mathrm{~F}^{\mathrm{O}}$ & - & $6 d^{2} D$ \\
\hline 1167.8 & & & Si VII & $3 s^{3} S_{1}^{o}$ & - & $3 p^{3} P_{2}$ \\
\hline 1168.85 & - & 1168.99 & C IV & $3 d^{2} D^{1}$ & - & $4 \mathrm{f}^{2} \mathrm{~F}^{\mathrm{o}}$ \\
\hline 1171.12 & - & 1172.00 & O VI & $4 \mathrm{p}^{2} \mathrm{P}^{\mathrm{o}}$ & - & $5 s^{2} S$ \\
\hline 1171.60 & & & Ne VIII & $6 \mathrm{~h}^{2} \mathrm{H}^{\mathrm{o}}$ & - & $8 \mathrm{i}^{2}$ I etc. \\
\hline 1183.85 & - & 1184.69 & Ne VIII & $5 d^{2} D$ & - & $6 \mathrm{p}^{2} \mathrm{P}^{\mathrm{o}}$ \\
\hline 1230.04 & - & 1230.52 & C IV & $3 \mathrm{p}^{2} \mathrm{P}^{\mathrm{o}}$ & - & $4 s^{2} S$ \\
\hline 1236.0 & & & Si VII & $3 s^{\prime 1} D^{o}$ & - & $3 p^{\prime 1} \mathrm{~F}$ \\
\hline 1238.82 & - & 1242.80 & $\mathrm{~N} \mathrm{~V}$ & $2 \mathrm{~s}^{2} \mathrm{~S}$ & - & $2 \mathrm{p}^{2} \mathrm{P}^{\mathrm{o}}$ \\
\hline 1548.20 & - & 1550.77 & C IV & $2 s^{2} S$ & - & $2 p^{2} \mathrm{P}^{\mathrm{o}}$ \\
\hline 1640.42 & & & He II & 2 & - & 3 \\
\hline 1932.04 & & & Ne VIII & $6 \mathrm{~h}^{2} \mathrm{H}^{\mathrm{o}}$ & - & $7 \mathrm{i}^{2} \mathrm{I}$ etc. \\
\hline 2253.39 & & & He II & 3 & - & 10 \\
\hline 2306.90 & & & He II & 3 & - & 9 \\
\hline 2386.13 & & & He II & 3 & - & 8 \\
\hline 2404.44 & - & 2405.20 & C IV & $4 p^{2} P^{o}$ & - & $5 d^{2} D$ \\
\hline 2511.96 & & & He II & 3 & - & 7 \\
\hline 2524.81 & - & 2525.06 & C IV & $4 d^{2} D$ & - & $5 f^{2} \mathrm{~F}^{o}$ \\
\hline 2530.77 & & & C IV & $4 \mathrm{f}^{2} \mathrm{~F}^{\mathrm{O}}$ & - & $5 \mathrm{~g}^{2} \mathrm{G}$ \\
\hline 2734.11 & & & He II & 3 & - & 6 \\
\hline 2821.53 & & & $\mathrm{Ne}$ VIII & $3 \mathrm{~s}^{2} \mathrm{~S}_{1 / 2}$ & - & $3 \mathrm{p}^{2} \mathrm{P}_{3 / 2}^{\mathrm{o}}$ \\
\hline 2860.94 & & & $\mathrm{Ne}$ VIII & $3 \mathrm{~s}^{2} \mathrm{~S}_{1 / 2}$ & - & $3 \mathrm{p}^{2} \mathrm{P}_{1 / 2}^{\mathrm{o}}$ \\
\hline 2906.93 & & & C IV & $5 g^{2} \mathrm{G}$ & - & $7 \mathrm{~h}^{2} \mathrm{H}^{\mathrm{o}}$ \\
\hline 2976.75 & & & Ne VIII & $7 \mathrm{i}^{2} \mathrm{I}$ & - & $8 \mathrm{k}^{2} \mathrm{~K}^{\mathrm{o}}$ etc. \\
\hline 2982.18 & & & $\mathrm{Nv}$ & $5 g^{2} G$ & - & $6 \mathrm{~h}^{2} \mathrm{H}^{\mathrm{o}}$ etc. \\
\hline
\end{tabular}

Notes. Wavelengths are from NIST, CHIANTI, and Kentucky databases with few exceptions (see Sect. 2).

results of our analysis are presented in Sect. 4. We conclude with a discussion of the evolutionary context in Sect. 5 .
Table 1. continued. Lines with $\lambda>3000 \AA$ (air wavelengths).

\begin{tabular}{|c|c|c|}
\hline Wavelength / A & Ion & Transition \\
\hline 3203.10 & He II & $3-5$ \\
\hline 3811.35 & O VI & $3 \mathrm{~s}^{2} \mathrm{~S}_{1 / 2}-3 \mathrm{p}^{2} \mathrm{P}_{3 / 2}^{\mathrm{o}}$ \\
\hline 3834.24 & OVI & $3 \mathrm{~s}^{2} \mathrm{~S}_{1 / 2}-3 \mathrm{p}^{2} \mathrm{P}_{1 / 2}^{\mathrm{o}}$ \\
\hline 4340.77 & Ne VIII & $8 \mathrm{k}^{2} \mathrm{~K}^{\mathrm{o}}-9 \mathrm{1}^{2} \mathrm{~L}$ etc. \\
\hline 4519.87 & $\mathrm{NV}$ & $7 \mathrm{i}^{2} \mathrm{I}-9 \mathrm{k}^{2} \mathrm{~K}^{\mathrm{o}}$ etc. \\
\hline 4541.59 & He II & 4-9 \\
\hline 4603.73 & $\mathrm{Nv}$ & $3 \mathrm{~s}^{2} \mathrm{~S}_{1 / 2}-3 \mathrm{p}^{2} \mathrm{P}_{3 / 2}^{\mathrm{o}}$ \\
\hline 4619.98 & $\mathrm{Nv}$ & $3 \mathrm{~s}^{2} \mathrm{~S}_{1 / 2}-3 \mathrm{p}^{2} \mathrm{P}_{1 / 2}^{\mathrm{o}}$ \\
\hline 4657.56 & CIV & $5 f^{2} \mathrm{~F}^{\mathrm{o}}-6 \mathrm{~g}^{2} \mathrm{G}$ etc. \\
\hline 4658.20 & C IV & $5 \mathrm{~g}^{2} \mathrm{G}-6 \mathrm{~h}^{2} \mathrm{H}^{\mathrm{o}}$ \\
\hline 4685.71 & He II & $3-4$ \\
\hline 4859.32 & He II & 4-8 \\
\hline 4933.90 & $\mathrm{NV}$ & $6 \mathrm{~d}^{2} \mathrm{D}-7 \mathrm{f}^{2} \mathrm{~F}^{\mathrm{o}}$ etc. \\
\hline 4944.56 & $\mathrm{Nv}$ & $6 \mathrm{~h}^{2} \mathrm{H}^{\mathrm{o}}-7 \mathrm{i}{ }^{2} \mathrm{I}$ etc. \\
\hline 5411.53 & He II & $4-7$ \\
\hline 5665. & $\mathrm{Ne}$ VII & $8 \mathrm{i}-9 \mathrm{k}$ \\
\hline 5669. & $\mathrm{Ne} \mathrm{VII}$ & $8 \mathrm{k}-91$ \\
\hline 6068.63 & Ne VIII & $91^{2} \mathrm{~L}-10 \mathrm{~m}^{2} \mathrm{M}^{\mathrm{o}}$ etc. \\
\hline 6560.10 & He II & 4-6 \\
\hline
\end{tabular}

\section{Observations and line identifications}

We utilised HST, FUSE, Calar Alto, and Keck spectroscopy. All datasets were described in our earlier publications cited in the introduction. In essence, the FUSE spectra cover the FUV range (912-1180 $\AA$ ) with a resolution of $0.05-0.1 \AA$. The HST spectra cover the UV range $(1150-3300 \AA)$ with different resolutions (0.1-1 $\AA$ ), depending on the detailed instrumental setup. In the optical range, we used spectra obtained with the Calar Alto $3.5 \mathrm{~m}$ telescope $(3350-6700 \AA)$ at resolutions of $1.5-3 \AA$ and Keck data (4270-6710 $)$ ) with $0.1 \AA$ resolution. We did inspect archival UV spectra obtained with IUE, ORFEUS, and HUT, but their quality is inferior.

All spectra were scrutinised for photospheric lines that have not been discovered or identified before. In many cases, this was done by looking in synthetic spectra for possible line candidates. Problems with imprecisely known line positions are described in some detail below. All identified photospheric lines are specified in Table 1. In summary, we see lines from the following metal ions: C IV, N v, O vI, Ne VII-VIII, Si VII, S VI-VII, CaX, and $\mathrm{Fe} \mathrm{X}$.

For a few lines, the observed positions deviate significantly from those given in the atomic databases (or those calculated from the given level energies). These are the discovered S VII line (see Sect. 2.3) and a pair of Fe X lines (Sect. 2.4), plus two Ca X lines (reported by Werner et al. 2008) in the FUSE spectrum, as well as two Ne VII lines in the optical $(\lambda \lambda 5665,5669 \AA$; Werner et al. 2007). We also note that a few O VI UV lines have positions that were empirically determined from observations of PG1159-035 (Jahn et al. 2007).

\subsection{Hydrogen and helium}

The spectrum of KPD $0005+5106$ is dominated by ionised helium lines, which are mostly broad and shallow except for the UV Balmer lines (Fig. 1). The He II $\lambda \lambda$ 4686, $6560 \AA$ lines exhibit prominent central emission reversals. The emission line near $\lambda 4339 \AA$ is not from He II but from Ne VIII. There is no hint of any features from the hydrogen Balmer lines (Fig. 2). 
D. Wassermann et al.: Metal abundances in the hottest known DO white dwarf (KPD 0005+5106)

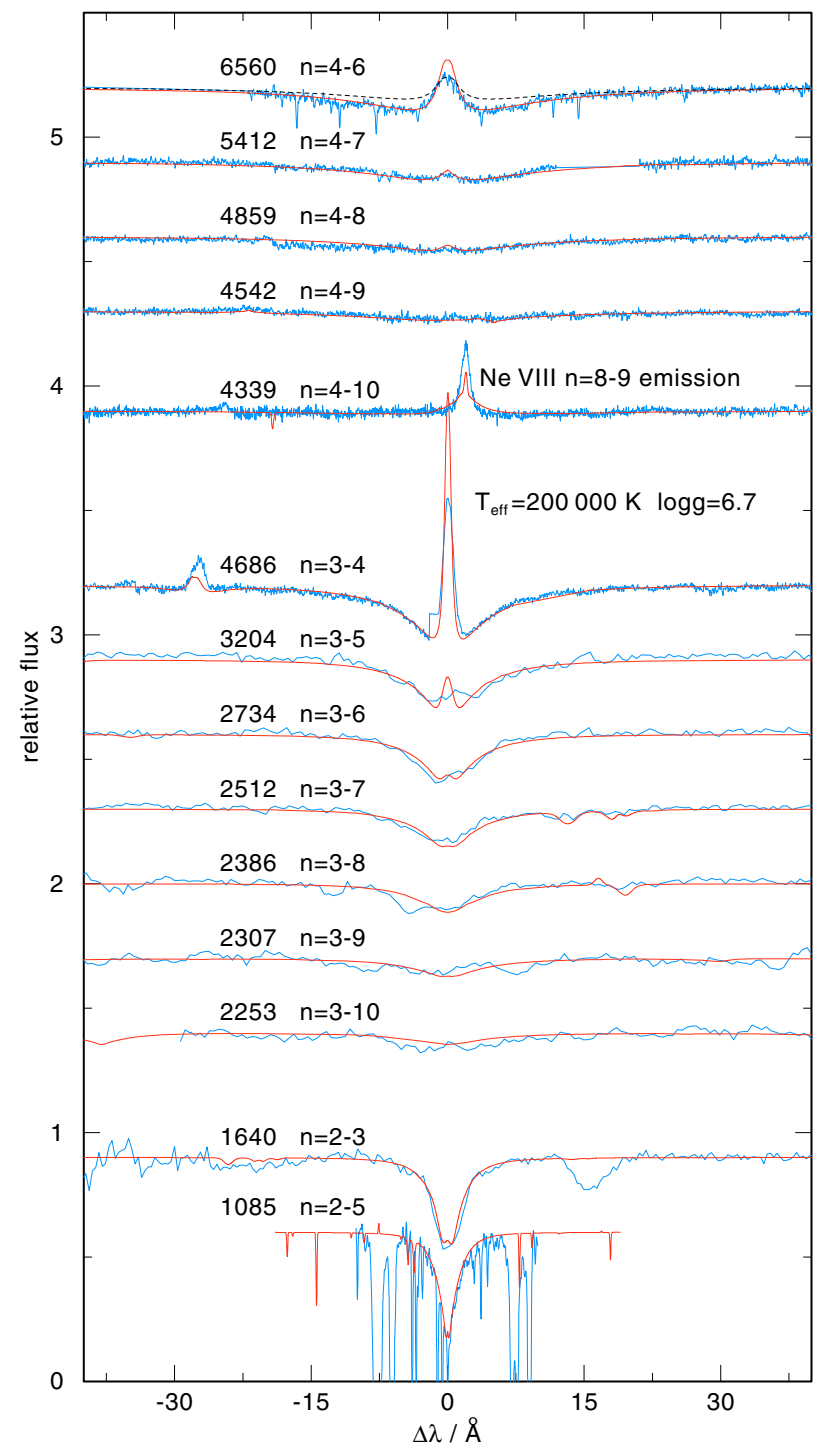

Fig. 1. Observed He II line profiles of the Pickering, Fowler, and Balmer series (from top to bottom) compared to a model with $T_{\text {eff }}=200000 \mathrm{~K}$ and $\log g=6.7$. The model includes metals with the finally adopted abundances. For comparison, the $\lambda 6560 \AA$ line (top) from a purehelium model is shown (dashed). The corresponding temperature structures are displayed in Fig. 11.

It turns out that fine-structure splitting of the $\lambda$ He II $4686 \AA$ line is large enough to have a detectable effect on the sharp Doppler emission-line core at the spectral resolution of the Keck spectrum. The individual components have a maximum separation of $0.54 \AA$, which is of the same order of magnitude as the Doppler width at $T=200000 \mathrm{~K}(0.50 \AA)$. The emission-peak height is slightly reduced, but it is still too high compared to the observation (Fig. 3). An additional broadening agent could be stellar rotation. When the line is folded with a rotation profile according to $v \sin i=52 \mathrm{~km} \mathrm{~s}^{-1}$, then the observation is matched much better. However, the emission-peak height is very sensitive to atmospheric parameters, in particular to the metal abundances.

\section{2. $\mathrm{C}, \mathrm{N}, \mathrm{O}, \mathrm{Ne}$, and $\mathrm{Ca}$}

A number of lines (well known from PG1159 stellar spectra) from lithium-like C, N, and O ions (C IV, N v, O VI; e.g. Miksa et al. 2002) is present (Figs.4-6). It is remarkable that a few

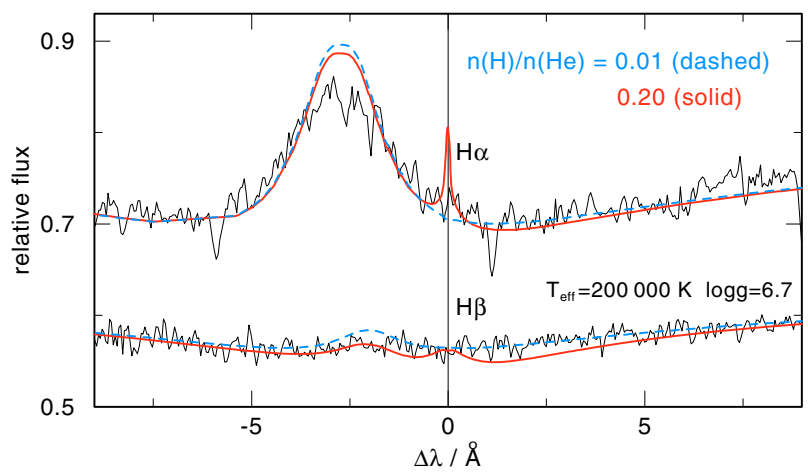

Fig. 2. He II line cores near the positions of $\mathrm{H} \alpha$ and $\mathrm{H} \beta$. Overplotted are two models with different hydrogen abundance. The more hydrogenrich model exhibits an unobserved emission core in $\mathrm{H} \alpha$ whereas it produces an unobserved depression in the range $\pm 5 \AA$ around the line centre of $\mathrm{H} \beta$.

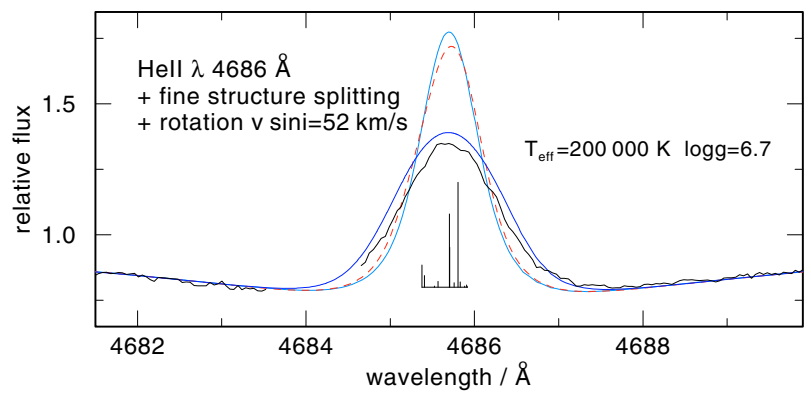

Fig. 3. Effect of fine-structure splitting (dashed line) and rotational broadening (thick full line) on the emission core of He II $\lambda 4686 \AA$ (thin full line; strongest emission core). To account for instrumental broadening, all model profiles were convolved with a Gaussian with $F W H M=0.1 \AA$. The positions of the individual line components are indicated by vertical bars, whose length is proportional to the $g f$-value. The data gap around $4684 \AA$ stems from incomplete coverage of Echelle orders.

other, usually prominent lines from these ions are not seen in KPD $0005+5106$. The complete lack of the emission feature of O VI $\lambda 5292 \AA$ and the C IV $\lambda \lambda 5801 / 5812 \AA$ emission doublet imposes strong constraints on the photospheric parameters (Sect. 4.1). We do not confirm Feibelman's (1999) identification of an O VII line at $\lambda 1522 \AA$ in IUE data. Our test calculations indicate that KPD $0005+5106$ is not hot enough to exhibit excited $\mathrm{O}$ VII lines. The existence of this feature is not confirmed by the higher quality FOS spectrum.

Several Ne VIII lines are found in the entire UV and optical wavelength regions (Fig. 7). Most of them have already been reported in Werner et al. (2007). We do not see Ne VII $\lambda 3644 \AA$, imposing a lower limit on $T_{\text {eff }}$. On the other hand, two weak Ne VII emission lines appear at $\lambda \lambda 5665 / 5669 \AA$; however, they stem from very highly excited levels, which are not included in our model atom.

The appearance of the CaX $\lambda \lambda 1137 / 1159 \AA$ doublet in KPD $0005+5106$ was reported earlier (Werner etal. 2008). Together with the Ne VIII lines, they point to very high $T_{\text {eff }}$.

\subsection{Silicon and sulphur}

We have identified lines of Si VII. This is the first discovery of such highly ionised silicon in a stellar photosphere. In the $\lambda 1132-1156 \AA$ range, we find six lines from the triplet and 


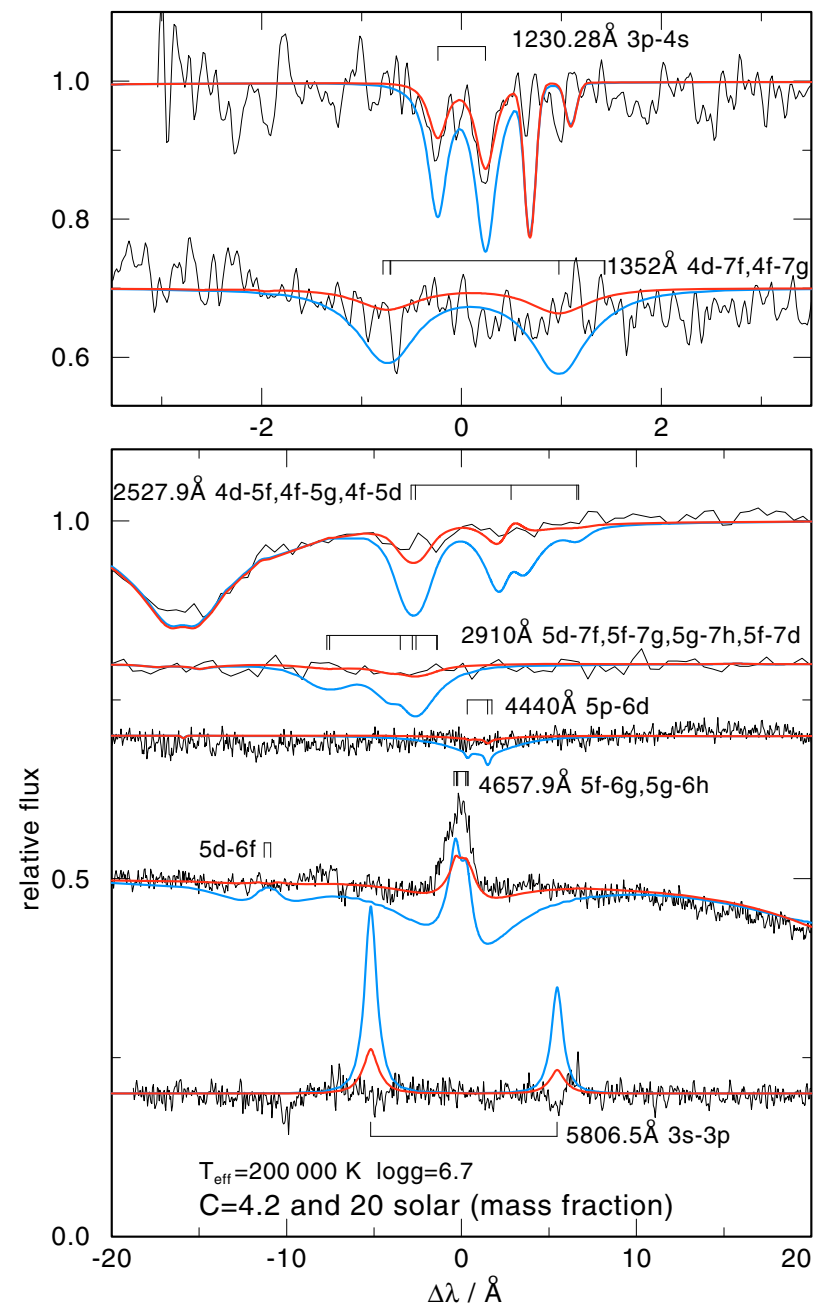

Fig. 4. Observed C IV lines and two models with different carbon abundances. Note the absence of the $3 \mathrm{~s}-3 \mathrm{p}$ doublet at $\lambda 5800 \AA$. The model with the higher carbon abundance exhibits much too strong emission lines. Here and in following figures: wavelengths given at line identifications correspond to $\Delta \lambda=0 \AA$.

quintet systems (Fig. 8), and a singlet at $\lambda 1236 \AA$. We do not see lines from the adjacent ionisation stages Si VI and Si VIII (Fig.9). This serves to confirm and constrain the very high $T_{\text {eff }}$ of KPD $0005+5106$.

The S VI $\lambda \lambda$ 933/944 A resonance doublet is strongly blended by interstellar lines. The $\lambda 944 \AA$ component is probably present. Another subordinate and unblended line of S VI at $\lambda 1118 \AA$ was discovered (Fig. 10). A search for weak S VII lines that are predicted by our models was positive $\left(3 \mathrm{~s}{ }^{3} \mathrm{P}^{\mathrm{o}}-3 \mathrm{p}{ }^{3} \mathrm{~S}\right.$ transitions at $\lambda \lambda 1051.54,1102.83,1175.4 \AA$; Kentucky database ${ }^{1}$ wavelengths). The first component was discovered at a slightly redshifted $(\approx 0.2 \AA)$ position (listed in Table 1$)$. The second and third components are weaker and are not identified in the observation. $S$ VIII lines are very weak in the models and cannot be detected in the FUSE spectra. With S VI/S VII we have another independent ionisation balance in hand to constrain $T_{\text {eff }}$.

\subsection{Iron}

KPD $0005+5106$ is much too hot to exhibit Fe VII lines. This confirms our earlier suspicion (Werner etal. 1996; Miksa etal. 2002) that the Fe VII $\lambda 1232.69 \AA$ line identified by

\footnotetext{
${ }^{1}$ http://www.pa.uky.edu/ peter/atomic/
}
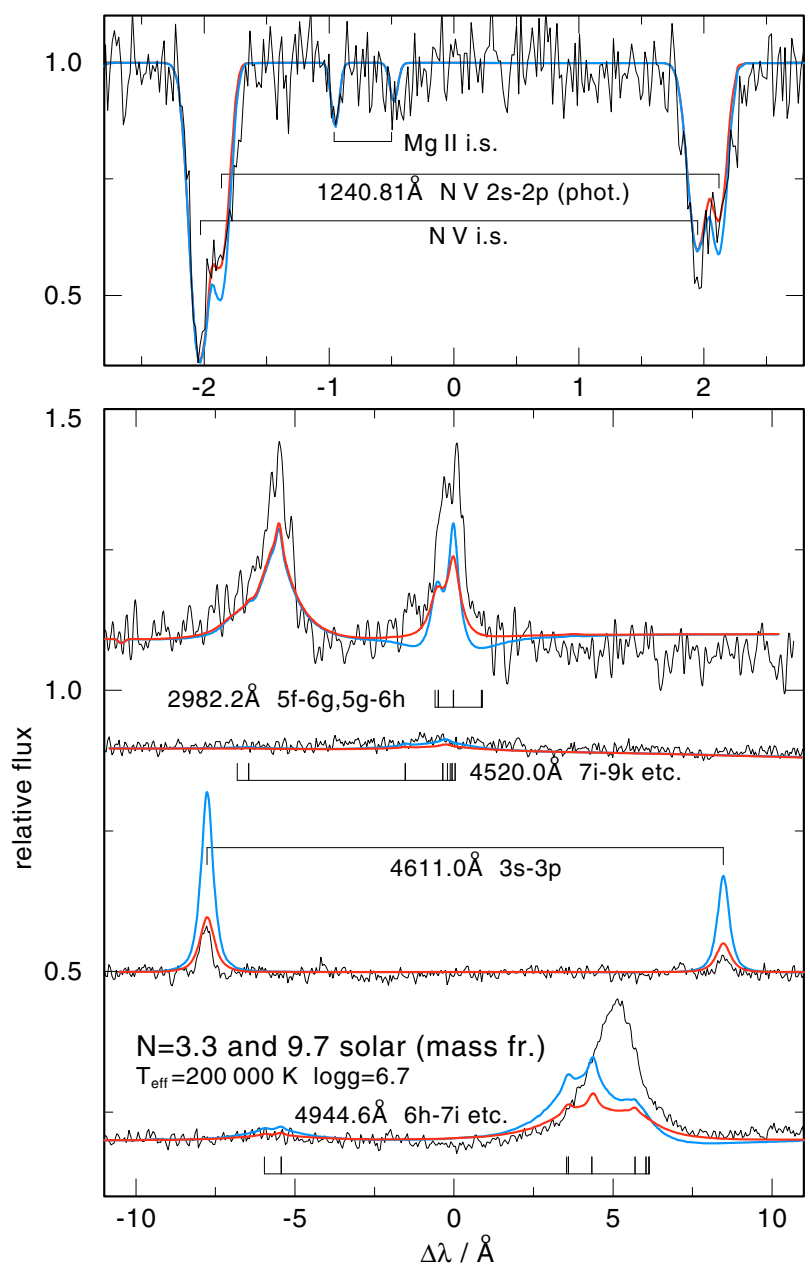

Fig. 5. Observed Nv lines and two models with different nitrogen abundances.

Feibelman \& Bruhweiler (1990) is in fact an interstellar Mg II line. Only two Fe IX lines from the Kurucz (2009) POS list $(\lambda \lambda 955.84,973.71 \AA)$ are predicted by our models to be potentially strong enough to be identified in KPD 0005+5106, but only if the abundance is roughly 1 dex oversolar. The former is the strongest but unfortunately, it is located in the wing of a strong blend of interstellar lines.

The iron ionisation structure (Fig. 11) shows that it is more promising to look for lines from Fe X and Fe XI, however, no Kurucz data are available for these ions. The Opacity Project (OP) and Iron Project (TIPTOPbase ${ }^{2}$, Seaton et al. 1994; Hummer et al. 1993) line lists used for our model calculations give only computed (and no observed) line positions, and the lines are not fine-structure split.

For $\mathrm{FeX}$ we found candidates from the Kentucky and CHIANTI (Dere etal. 1997, 2009) databases. They are three lines from a transition in the doublet system, located at $\lambda \lambda$ 980.2, 1017.9, and 1023.1 $\AA$. Their positions are uncertain by $0.9 \AA$, according to the Kentucky database. The two strongest components $(\lambda \lambda 980.2$ and $1023.1 \AA$ ) have high $g f$-values (1.90 and 1.33). Within the uncertainty range, there are two strong features in the FUSE spectrum (at $\lambda \lambda 979.3$ and 1022.9 $\AA$ ) that we identify with these lines (Fig. 12, Table 1). We can calculate the true fine-structure splitting of the energy levels from the two

${ }^{2}$ http://vizier.u-strasbg.fr/topbase/ 


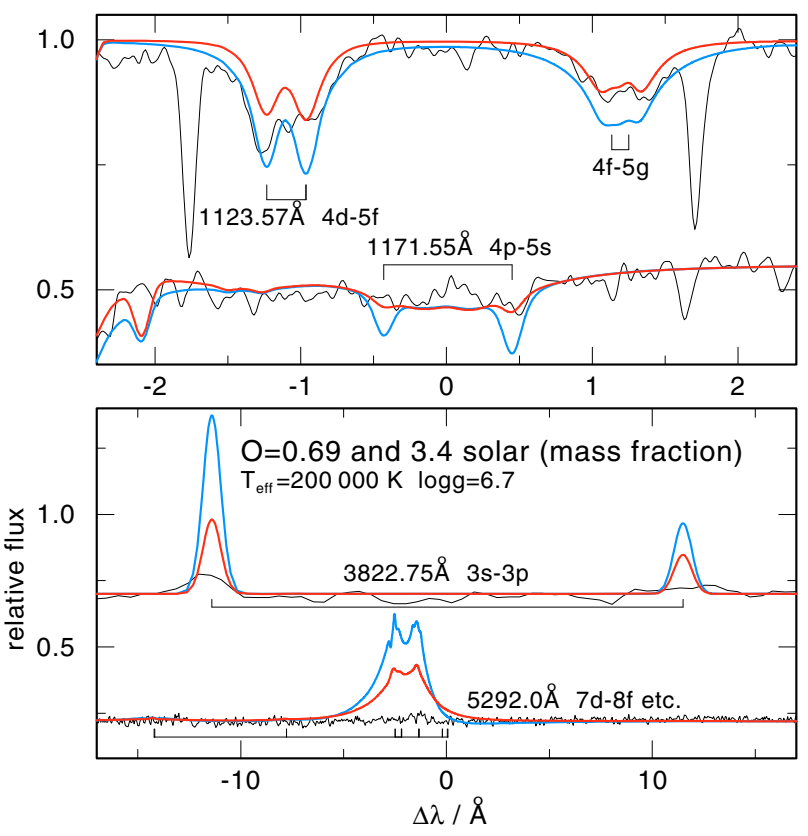

Fig. 6. Observed OVI lines and two models with different oxygen abundances.

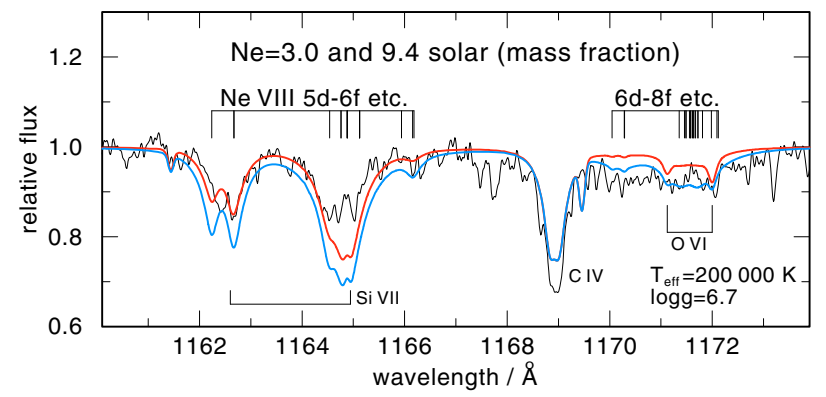

Fig. 7. Observed Ne VIII lines and two models with different neon abundances.

observed lines' positions and determine the expected position of the third component (which is much weaker; $g f=0.095$ ). It is almost $1 \AA$ blueward of the listed position: $\lambda 1016.95 \AA$. In the spectrum of KPD $0005+5106$ this is located in the wing of an interstellar $\mathrm{H}_{2}$ line and cannot be detected.

For our spectrum calculation, we used the oscillator strength of this doublet transition from the OP database. The $g f$ values of the fine-structure split lines were calculated according to quantum-mechanical prescription. Our empirically derived level energies related to this particular Fe X transition represent an improvement of the atomic databases:

$$
\begin{array}{ll}
4 \mathrm{~s}^{2} \mathrm{D}_{5 / 2} & 1063596 .+\mathrm{x} \mathrm{cm} \\
4 \mathrm{~s}^{2} \mathrm{D}_{3 / 2} & 1064169 .+\mathrm{x} \mathrm{cm} \\
4 \mathrm{p}^{2} \mathrm{~F}_{5 / 2}^{\mathrm{o}} & 1161930 .+\mathrm{x} \mathrm{cm} \\
4 \mathrm{p}^{-1} \\
{ }^{2} \mathrm{~F}_{7 / 2}^{\mathrm{o}} & 1165710 .+\mathrm{x} \mathrm{cm}
\end{array}
$$

We also discovered the Fe X lines in the hottest PG1159 stars, which for the first time enables us to determine the iron abundance in this spectral class (Werner et al. 2010).

\subsection{Unidentified photospheric lines}

A few, probably photospheric lines remain unidentified (Table 2). In the UV some sulphur or silicon lines with uncertain wavelength positions appear in our synthetic spectra for which we fail to identify a counterpart in the observation. The two unidentified optical emission lines could stem from highly excited Ne VII or Ne VIII Rydberg states with unknown level energies.

\section{Model atmospheres and synthetic spectra}

\subsection{Course of analysis}

We used the Tübingen Model Atmosphere Package TMAP (Werner et al. 2003; Rauch \& Deetjen 2003) to compute planeparallel non-LTE line-blanketed model atmospheres in radiative and hydrostatic equilibrium. The models include all the elements ever discovered in KPD 0005+5106 except iron, plus hydrogen (i.e. $\mathrm{H}, \mathrm{He}, \mathrm{C}, \mathrm{N}, \mathrm{O}, \mathrm{Ne}, \mathrm{Si}, \mathrm{S}, \mathrm{Ca}$ ). Table 3 summarises the utilised model atoms. For computational reasons, the model atmosphere structure was computed with somewhat smaller model atoms for nitrogen and calcium. N VIII was omitted and N VII represented by its ground state only, and Ca X had 15 NLTE levels linked by 44 lines. Numbers of NLTE levels and lines for silicon ions were $(15,20),(20,36)$ for Si V,VI, respectively. The larger versions of these model atoms as listed in Table 3 were subsequently used to further iterate the atomic population numbers, keeping fixed the atmospheric temperature and pressure structure. Iron was not included in the model computations. Its NLTE level populations were then computed for a fixed model structure.

The computing time for a single model atmosphere is too long to compute an extensive grid with $T_{\text {eff }}, \log g$, and element abundances as parameters. We first computed a grid of purehelium models with different $T_{\text {eff }}$ and $\log g$ in order to achieve a fit to the He II lines. We then performed an element-by-element study by including one additional species in the helium models to derive a first estimate for the metal abundances. In this step we also checked whether the metal lines give additional constraints to $T_{\text {eff }}$ and $\log g$ by inspecting ionisation equilibria. Then a small grid of full model atmospheres (including all metals with a particular abundance pattern) with different $T_{\text {eff }}$ and $\log g$ was computed in the vicinity of the finally adopted values $200000 \mathrm{~K}$ and 6.7, respectively. We then made fine adjustments to these parameters by cross-checking the He II and metal line fits and adjusting the individual element abundances.

In Fig. 11 we display the ionisation structure of the model with the finally adopted parameters, as well as the run of temperature. For comparison we show the temperature structure of a pure-helium model, which has a high-temperature plateau in the outer layers. The influence of the metals by line blanketing and surface cooling are very strong and justify the computationally expensive NLTE modelling. As an example, we compared the He II $\lambda 6560 \AA$ line of our final model to that of a pure-helium model (Fig. 1) and realise that the latter profile is much too shallow to fit the observation.

An unexpected numerical problem occurred with our model atmosphere code. Unrealistic upward and downward spikes in the form of a narrow hysteresis curve occurred around a particular frequency point on the red and blue sides of the emission core of the He II $\lambda 6560 \AA$ line (and to a less significant extent in the weaker emission cores of He II $\lambda \lambda 5412,4859 \AA$ ). That effect can escape attention when the frequency grid is coarse enough, such that this hysteresis feature is not sampled. Close inspection revealed that the problem originates from the radiation transfer solver that, under certain circumstances, runs into numerical problems when the total opacity becomes negative between two adjacent depth points. The problem arises regardless of the 


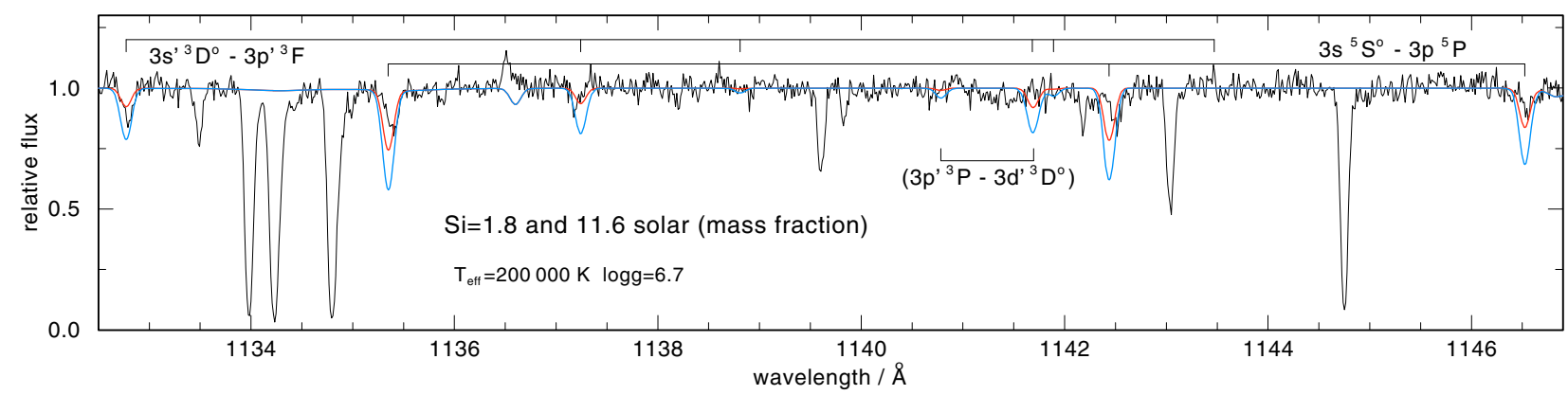

Fig. 8. Observed Si VII multiplets and two models with different silicon abundances. Some components of a triplet and a quintet transition are identified (top identification bars). Two weak components of another modelled triplet transition (lower identification bar) are not detectable in the observation. Other absorption lines have interstellar origins.

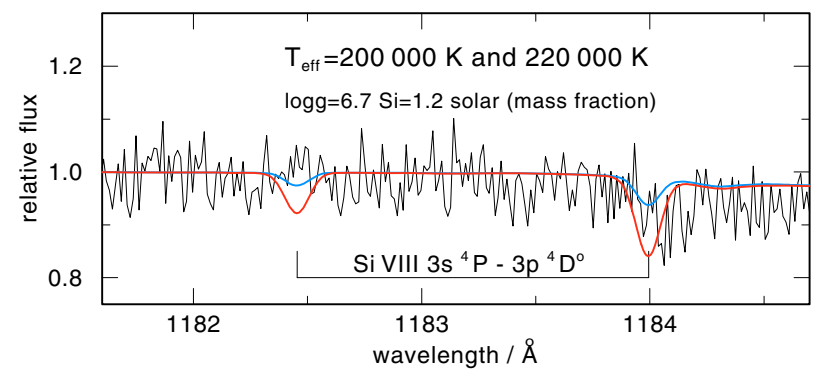

Fig. 9. The absence of Si VIII lines puts an upper limit to $T_{\text {eff }}$. We show two lines of a strong multiplet from two models with different $T_{\text {eff }}$. The hotter model shows deeper lines. They are not detectable in the FUSE spectrum. The depression at $\lambda>1184 \AA$ is due to broad Ne VIII lines.

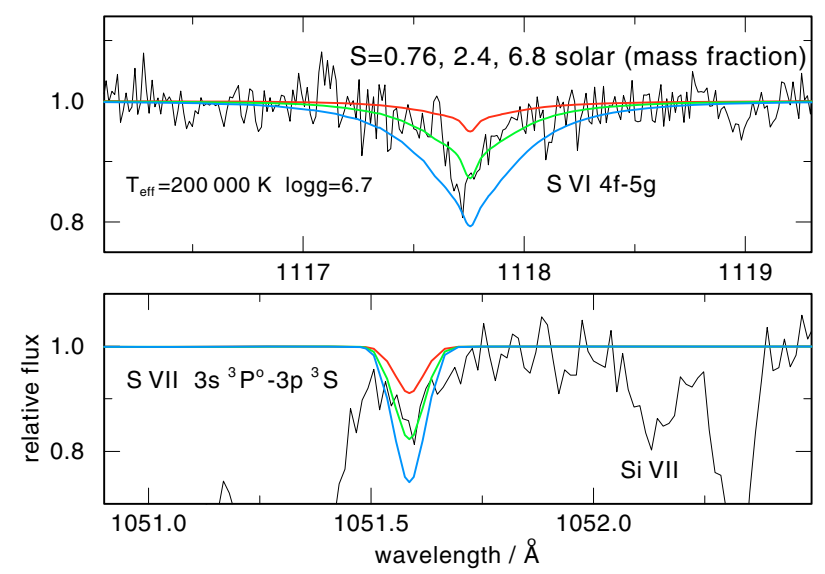

Fig. 10. Observed S VI (top panel) and S VII (bottom) lines and profiles from three models with different sulphur abundance.

solution technique used, whether a short-characteristic method or a Feautrier method. We suspect that the problem can be solved with an improved Feautrier scheme that leads to better numerical conditioning for small optical depth increments (Rybicki \& Hummer 1991).

A simple way to work around this problem is to refine the depth grid of the model atmosphere and then to perform a final formal solution of the transfer equation. To do this, we refined our depth grid from 90 to 1000 points with a linear interpolation of all population densities and temperature, and the problem disappeared completely.
Table 2. Measured wavelength positions of unidentified lines, possibly of photospheric origin.

\begin{tabular}{|c|c|c|}
\hline Wavelength / A & & Possible identification \\
\hline 990.66 & absorption & \\
\hline 1004.54 & absorption & Si VII $1005.1 \AA$ \\
\hline 1054.79-1054.86 & absorption & \\
\hline 1097.29-1097.52 & absorption & Si VII $1097.1 \AA$ \\
\hline 4296. & $\operatorname{emission}^{a}$ & \\
\hline 4314. & emission $^{b}$ & Ne VIII $8 f^{4} F^{o}-9 g{ }^{4} G$ \\
\hline
\end{tabular}

Notes. ${ }^{(a)}$ Absorption line in hot PG1159 stars.

(b) Also seen in H1504+65.

\subsection{Model atoms}

Model atoms for most species were taken over from previous work. Those for $\mathrm{H}, \mathrm{He}, \mathrm{C}, \mathrm{N}$, and $\mathrm{O}$ were described, e.g. in Jahn et al. (2007). CNO models were extended to higher ionisation stages in order to obtain correct ionisation equilibria. For $\mathrm{Ne}$ and Ca models see Werner et al. (2007, 2008). Generally, finestructure splitting of energy levels and detailed line broadening is accounted for in the final formal solution for the radiation transfer equation, by which the synthetic spectra are obtained.

Strong effort in the present context was necessary for the new construction of high ionisation stages of sulphur and, in particular, silicon model atoms, because of the discovery of several Si VII lines in the FUSE spectra. Figure 13 is a Grotrian diagram of our Si V-VIII model which, together with the information given in Table 1, allows us to locate the investigated line transitions.

As sources for atomic data, we used the atomic databases of NIST ${ }^{3}$ and CHIANTI for the energy levels and the OP and Iron Project for the oscillator strengths. The Kentucky database was used for spectral line positions and oscillator strengths. The energy levels and line lists from Kurucz (2009) were used for the iron model ions Fe VII-IX.

We must refrain here from a detailed description of the design of the model atoms. Many test calculations were performed, e.g., in order to decide which f-values are selected from which database. However, all our model atoms are publicly available. They were developed within the framework of the German Astrophysical Virtual Observatory $\left(\mathrm{GAVO}^{4}\right)$ project and are staged in the Tübingen Model-Atom Database TMAD ${ }^{5}$.

\footnotetext{
3 http://www.nist.gov/physlab/data/asd.cfm

4 http://www.g-vo.org

5 http://astro.uni-tuebingen.de/ rauch/TMAD/TMAD.html
} 
D. Wassermann et al.: Metal abundances in the hottest known DO white dwarf (KPD 0005+5106)
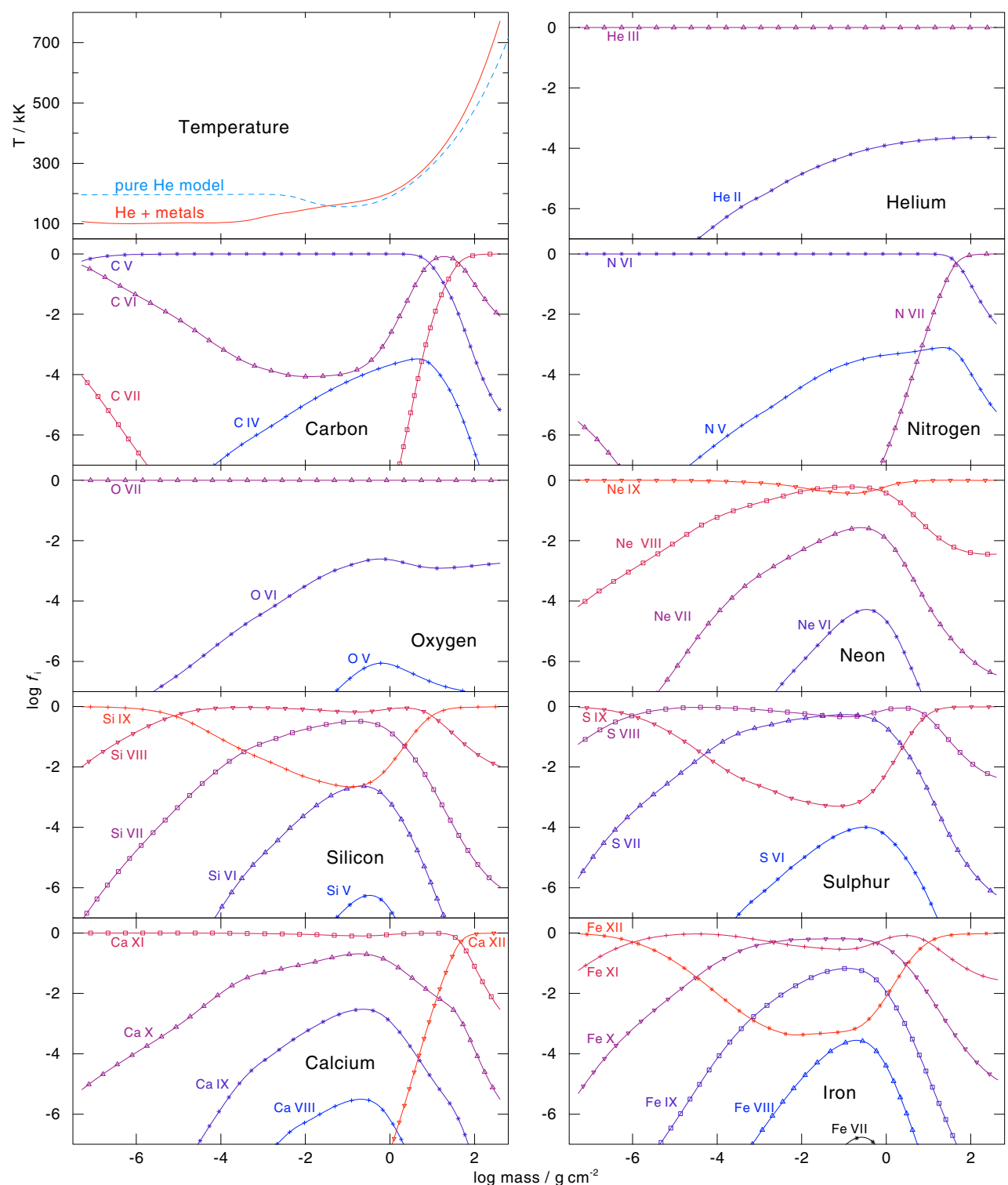

Fig. 11. Vertical run of temperature (top left panel) and ionisation fractions $f_{\mathrm{i}}$ of elements in a model with $T_{\text {eff }}=200000 \mathrm{~K}$ and $\log g=6.7$. The temperature structure of a pure-helium model is shown for comparison.

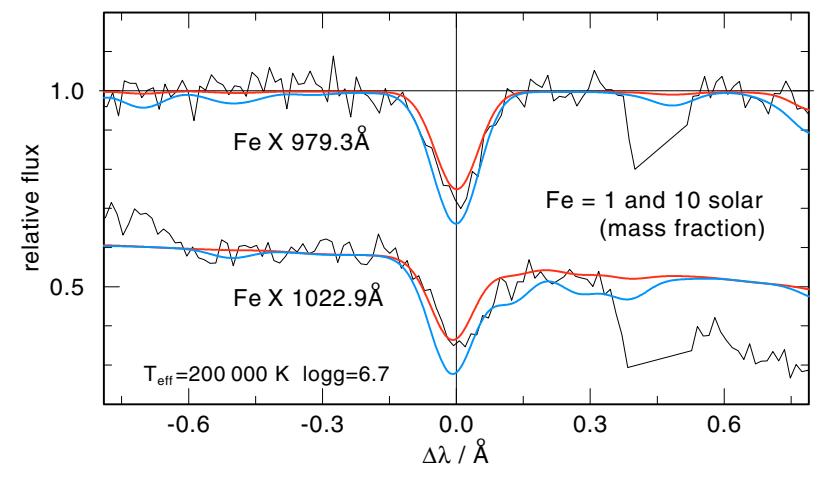

Fig. 12. Observed Fe $X$ lines and two models with different Fe abundances. A strong ISM line is cut out at $\Delta \lambda=0.5 \AA$.

\section{Results}

The results of our analysis are summarised in Tables 4 and 5.

\subsection{Effective temperature and surface gravity}

There is not a single model that perfectly fits all line features simultaneously. We adopted error estimates for $T_{\text {eff }}(10 \%)$ and $\log g( \pm 0.3$ dex $)$ such that we did find a model that can reproduce the spectral lines of different elements in the respective parameter range. For example, to have the $\mathrm{CaX}$ lines in emission the model needs to have a relatively low $\log g(6.5$ at $\left.T_{\text {eff }}=200000 \mathrm{~K}\right)$. On the other hand, the lack of the emission features of O VI at $\lambda 5292 \AA$ and C IV $\lambda 5801 / 5812 \AA$ requires a model with relatively low $T_{\text {eff }}$ (about $180000 \mathrm{~K}$ at $\log g=6.7$ ). The presence of Ne VIII lines over the entire UV and optical bands and the lack of Ne VII $\lambda 3644 \AA$ initiated our revision of $T_{\text {eff }}$ from $120000 \mathrm{~K}$ to $200000 \mathrm{~K}$, as already mentioned.

Silicon turned out to be another independent $T_{\text {eff }}$ indicator. The presence of Si VII lines and the absence of any line of the adjacent ionisation stages excludes temperatures below $180000 \mathrm{~K}$ and above $220000 \mathrm{~K}$ (Figs. 8, 9). The sulphur ionisation balance is even more useful, because $\mathrm{S}$ VI and S VII lines are detected (Fig. 10). The best fit is obtained at $T_{\text {eff }}=200000 \mathrm{~K}$. Increasing or decreasing $T_{\text {eff }}$ by $10 \%$ results in S VI or S VII 
A\&A 524, A9 (2010)
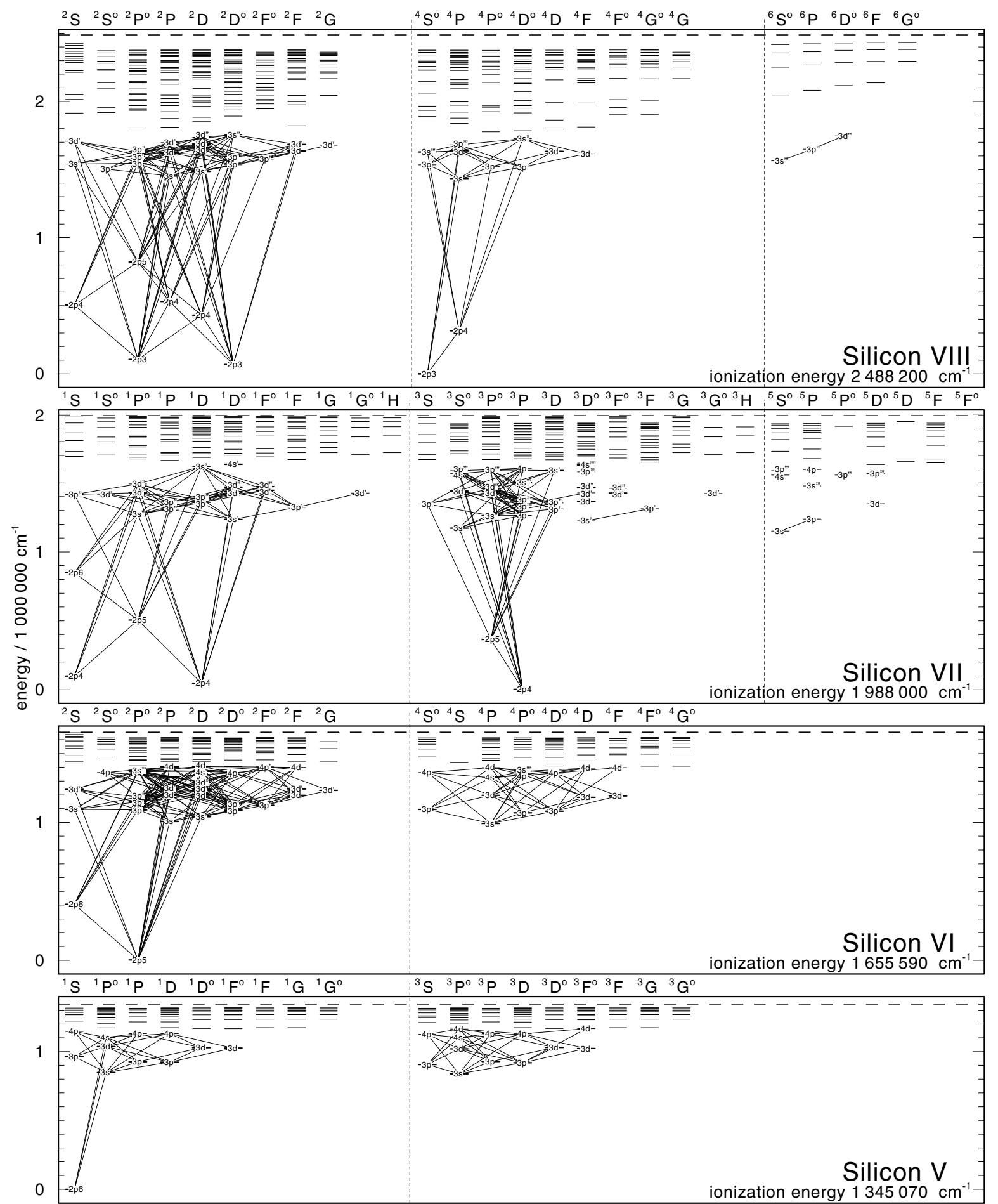

Fig. 13. Grotrian diagrams of our model ions for Si V-VIII. All levels that are linked by line transitions are treated in NLTE, others in LTE. Thick drawn are levels with energies from NIST, all others are from OP.

lines, respectively, that are too weak. There is a dependence of the metal ionisation balances, hence their line strengths, on gravity. This was accounted for by constraining $T_{\text {eff }}$ and $\log g$ from the fit to the He II lines.

\subsection{Abundances}

We varied the metal abundances in a $T_{\text {eff }} / \log g$ grid of models within the error ranges of these parameters. The effect of these abundance variations on the line profiles is exemplified for carbon (Fig. 4), nitrogen (Fig. 5), oxygen (Fig. 6), neon (Fig. 7), silicon (Fig. 8), sulphur (Fig. 10), and iron (Fig. 12). For a detailed study of the CaX lines see Werner et al. (2008). The resulting abundances are listed in Table 5. We estimate the errors to be in the order of $0.5 \mathrm{dex}$, except for calcium. The Ca X emission lines are most sensitive to $T_{\text {eff }}$ and $\log g$ such that an error of 1 dex must be accepted. To significantly reduce the uncertainties we would primarily need to put a tighter constraint on $T_{\text {eff }}$. This could be achieved with high-resolution UV spectra in the 
D. Wassermann et al.: Metal abundances in the hottest known DO white dwarf (KPD 0005+5106)

Table 3. Summary of model atoms used in the NLTE calculations.

\begin{tabular}{|c|c|c|c|}
\hline Element & Ion & NLTE levels & Lines \\
\hline \multirow[t]{2}{*}{$\mathrm{H}$} & $\mathrm{I}$ & 5 & 10 \\
\hline & II & 1 & - \\
\hline \multirow[t]{3}{*}{$\mathrm{He}$} & I & 5 & 3 \\
\hline & II & 16 & 119 \\
\hline & III & 1 & - \\
\hline \multirow[t]{4}{*}{$\mathrm{C}$} & IV & 54 & 295 \\
\hline & $\mathrm{V}$ & 41 & 95 \\
\hline & VI & 55 & 288 \\
\hline & VII & 1 & - \\
\hline \multirow[t]{3}{*}{$\mathrm{N}$} & $\mathrm{V}$ & 54 & 297 \\
\hline & VI & 36 & 164 \\
\hline & VII & 1 & - \\
\hline \multirow[t]{3}{*}{$\mathrm{O}$} & $\mathrm{V}$ & 90 & 610 \\
\hline & VI & 54 & 291 \\
\hline & VII & 1 & - \\
\hline \multirow[t]{5}{*}{$\mathrm{Ne}$} & $\mathrm{V}$ & 30 & 69 \\
\hline & VI & 25 & 73 \\
\hline & VII & 103 & 761 \\
\hline & VIII & 77 & 510 \\
\hline & IX & 1 & - \\
\hline \multirow[t]{6}{*}{$\mathrm{Si}$} & IV & 16 & 44 \\
\hline & $\mathrm{V}$ & 15 & 20 \\
\hline & VI & 20 & 36 \\
\hline & VII & 61 & 138 \\
\hline & VIII & 41 & 147 \\
\hline & IX & 1 & - \\
\hline \multirow[t]{5}{*}{ S } & $\mathrm{V}$ & 10 & 8 \\
\hline & VI & 18 & 48 \\
\hline & VII & 38 & 120 \\
\hline & VIII & 24 & 24 \\
\hline & IX & 1 & - \\
\hline \multirow[t]{5}{*}{$\mathrm{Ca}$} & VIII & 1 & - \\
\hline & IX & 15 & 23 \\
\hline & $\mathrm{x}$ & 15 & 44 \\
\hline & XI & 4 & 2 \\
\hline & XII & 1 & - \\
\hline \multirow[t]{7}{*}{$\mathrm{Fe}$} & VI & (3165) 7 & 25 (840065) \\
\hline & VII & 7 & (86504) 24 \\
\hline & VIII & (310) 7 & 27 (8724) \\
\hline & IX & (712) 7 & (36843) 25 \\
\hline & $\mathrm{X}$ & (761) 7 & 28 (45 229) \\
\hline & XI & (546) 7 & 28 (29073) \\
\hline & XII & 1 & - \\
\hline Total: 10 & 43 & 975 & 4396 \\
\hline
\end{tabular}

Notes. All elements except of iron were included in the line-blanketed models. For iron we used a statistical superlevel/superline approach in the subsequent NLTE level population calculations. The original number of levels and lines are given in brackets.

wavelength range accessible to HST, where we expect many more weak metal lines of different ionisation stages.

\subsection{Mass, luminosity, distance}

The position of KPD $0005+5106$ in the $T_{\text {eff }} / \log g$ diagram is shown in Fig. 14. We derived stellar mass, radius, and luminosity (see Table 4) from a comparison with evolutionary tracks for DO white dwarfs taken from Althaus et al. (2009). The star is about to enter the WD cooling sequence. Strictly speaking, it is still a pre-WD: About $80 \%$ of its luminosity is generated by heliumshell burning.
Table 4. Derived parameters and distance of KPD 0005+5106.

\begin{tabular}{cc}
\hline \hline$T_{\text {eff }} / \mathrm{K}$ & $200000 \pm 20000$ \\
$\log \left(\mathrm{g} / \mathrm{cm} \mathrm{s}^{-2}\right)$ & $6.7 \pm 0.3$ \\
$M / M_{\odot}$ & $0.64_{-0.04}^{+0.08}$ \\
$R / R_{\odot}$ & $0.059_{-0.018}^{+0.031}$ \\
$\log L / L_{\odot}$ & $3.71_{-0.24}^{+0.48}$ \\
$d / \mathrm{pc}$ & $581_{-170}^{+2440}$ \\
\hline
\end{tabular}

Table 5. Element abundances in KPD $0005+5106$ given in various units: number ratio relative to helium $\mathrm{n}_{\mathrm{X}} / \mathrm{n}_{\mathrm{He}}$, mass fractions $\beta_{\mathrm{X}}$, and mass fractions in solar units $\beta_{\mathrm{X}} / \beta_{\odot}$.

\begin{tabular}{cccccc}
\hline \hline & $\log n_{\mathrm{X}} / n_{\mathrm{He}}$ & $\log \beta_{\mathrm{X}}$ & $\beta_{\mathrm{X}} / \beta_{\odot}$ & $\log \beta_{\odot}$ & $\log \beta_{\mathrm{X}}(\mathrm{RCrB})$ \\
\hline $\mathrm{H}$ & $<-1$ & $<-1.6$ & $<0.034$ & -0.13 & $\leq-5.2$ \\
$\mathrm{He}$ & 0.00 & -0.01 & 3.9 & -0.60 & -0.008 \\
$\mathrm{C}$ & -2.5 & -2.0 & 4.3 & -2.63 & -2.6 \\
$\mathrm{~N}$ & -3.2 & -2.6 & 3.3 & -3.16 & -2.4 \\
$\mathrm{O}$ & -3.0 & -2.4 & .69 & -2.24 & -2.7 \\
$\mathrm{Ne}$ & -3.1 & -2.4 & 3.0 & -2.90 & -2.5 \\
$\mathrm{Si}$ & -3.8 & -2.9 & 1.8 & -3.18 & -3.6 \\
$\mathrm{~S}$ & -4.0 & -3.1 & 2.4 & -3.51 & -3.8 \\
$\mathrm{Ca}$ & -4.6 & -3.6 & 3.6 & -4.19 & -5.2 \\
$\mathrm{Fe}$ & -4.0 & -2.9 & 1.0 & -2.89 & -3.9 \\
\hline
\end{tabular}

Notes. Solar abundances were adopted from Asplund et al. (2009). For comparison, the last column gives abundances in the majority $\mathrm{RCrB}$ stars. For error estimates see Sects. 4.2 and 5.

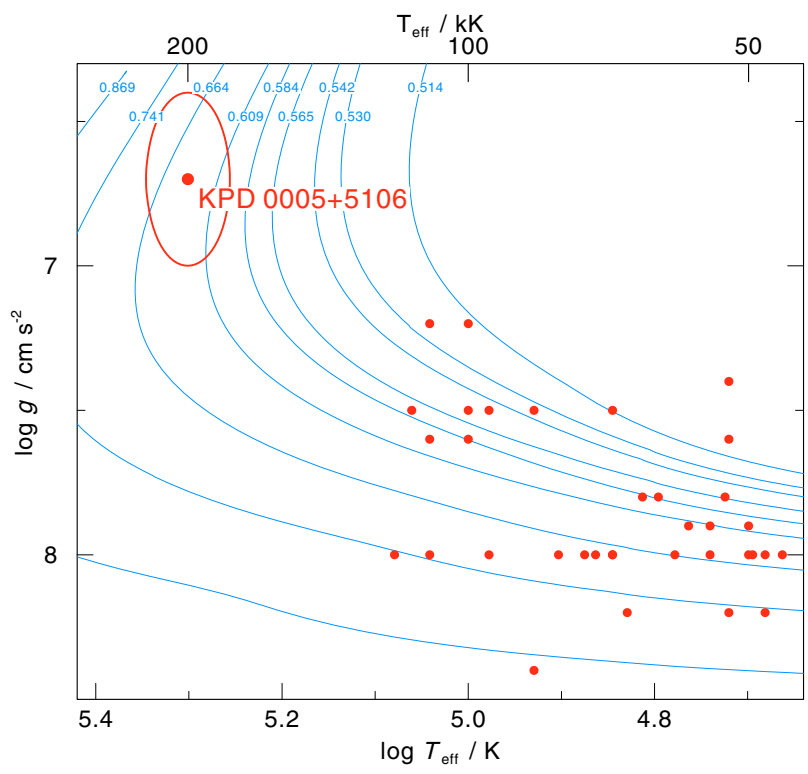

Fig. 14. Location of KPD $0005+5106$ in the $\log g-\log T_{\text {eff }}$ diagram together with all other known DO white dwarfs. The evolutionary tracks for H-deficient WDs are from Althaus et al. (2009). They are labeled with the respective masses in solar units.

The spectroscopic distance was estimated from

$d / \mathrm{pc}=6.6175 \times 10^{-6} \sqrt{F_{\gamma} / F_{\mathrm{obs}} \cdot M / M_{\odot} \cdot 10^{-\log g}}$

using the derived mass, where $F_{v}$ is the astrophysical flux at the surface of the star taken from the synthetic spectrum, and $F_{\text {obs }}$ is the observed flux after correction for extinction. Extinction was determined by a comparing the synthetic photometry derived from the model spectrum with the observed 


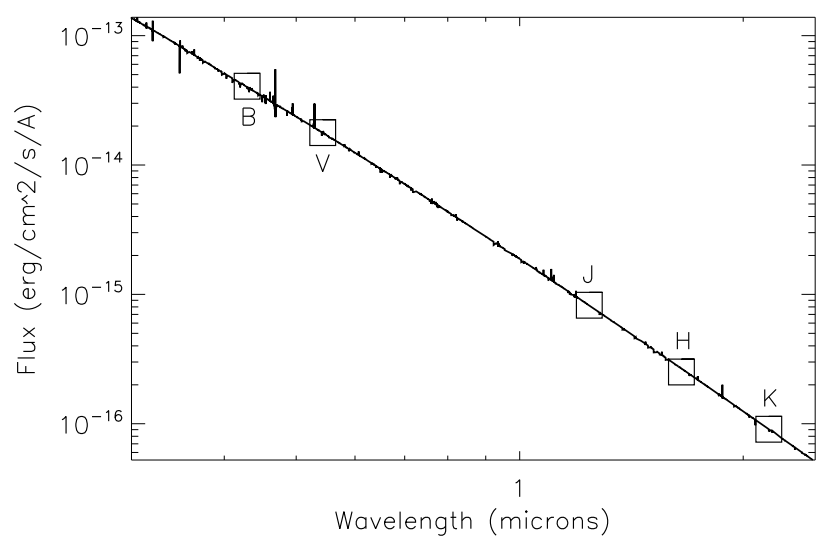

Fig. 15. Extinction-corrected synthetic spectrum and broad-band photometry.

Table 6. Broad-band photometry for KPD $0005+5106$.

\begin{tabular}{ccccc}
\hline \hline Band & $m_{\mathrm{obs}}$ & $A(\lambda)$ & $m_{\text {corr }}$ & $d / \mathrm{pc}$ \\
\hline$B$ & 13.020 & 0.461 & 12.559 & 574.9 \\
$V$ & 13.320 & 0.326 & 12.994 & 582.4 \\
$J$ & 13.948 & 0.084 & 13.864 & 578.5 \\
$H$ & 14.135 & 0.053 & 14.082 & 598.0 \\
$K_{\mathrm{s}}$ & 14.186 & 0.034 & 14.152 & 571.3 \\
\hline
\end{tabular}

visible (Downes et al. 1985) and 2MASS (Skrutskie et al. 2006) NIR photometry; the resulting parameters were $E(B-V)=0.124$ and $R_{V}=2.59$. Zero points for the visible and NIR magnitudes were taken from Cohen et al. (2003a) and Cohen et al. (2003b), respectively.

The extinction $A(\lambda)$ was then calculated with the formalism of Cardelli et al. (1989). Essentially identical results were obtained using the mean extinction curve given by Fitzpatrick \& Massa (2007; hereafter FM07), as amended in the NIR by Fitzpatrick \& Massa (2009). Table 6 gives the measured and extinction-corrected magnitudes, and the spectroscopic distance computed from the flux in each band. Extinction in each band (Col. 3) was obtained by fitting synthetic photometry derived from the model spectrum to the observed magnitudes (Col. 2). Corrected magnitudes are in Col. 4, and the corresponding spectroscopic distances are in Col. 5. The mean spectroscopic distance is $581 \mathrm{pc}$. The extinction-corrected synthetic spectrum and broad-band photometry are plotted in Fig. 15.

These extinction parameters provide a good fit to the broadband photometry, but overestimate the extinction in the UV. A fit to the entire spectral energy distribution, from the NIR through the FUV, yields extinction parameters of $R_{V}=3.0$ and $E(B-V)=0.07$. These parameters give a slightly greater distance of 600 pc. However, as discussed in detail by FM07, the parameters describing the UV extinction are poorly correlated with those describing the optical-NIR extinction. As we are interested in obtaining an estimate of the distance and not in the extinction itself, we have restricted this analysis to opticalNIR wavelengths. This has the advantage that the extinction is smaller, resulting in less sensitivity to measurement uncertainties, and the shape of the extinction curve varies less from one sightline to another (see, e.g., Fig. 15 of FM07).

\section{Summary and conclusions}

We have determined the photospheric parameters of KPD 0005+5106 using all relevant spectroscopic UV and optical observations in concert with line-blanketed non-LTE model atmospheres. We confirm the extremely high effective temperature and relatively low surface gravity. This clearly separates KPD 0005+5106 from all other known DO white dwarfs. Strictly speaking, it is a pre-white dwarf, located before the "knee" in the HRD. It is still helium-shell burning and has not yet reached its maximum $T_{\text {eff }}$. We determined the element abundance pattern. All metals are trace elements in a helium-dominated atmosphere that is devoid of hydrogen.

Effective temperature and luminosity of KPD $0005+5106$ are so high that a very weak radiation driven wind prevents gravitational settling of heavy elements in the atmosphere (Unglaub \& Bues 2000). It therefore must have descended from the AGB as an object with an already helium-dominated surface. It is possible that KPD $0005+5106$ could be a descendant of the RCrB stars, which are relatively cool post-AGB objects.

For RCrB stars two origins for their H-deficiency have been discussed in the literature for many years (e.g. Clayton 1996; de Marco et al. 2002). Either they are the result of a late thermal pulse (LTP) or of a merging process of two white dwarfs (double-degenerate scenario). Saio \& Jeffery (2002) have established the $\mathrm{CO}+\mathrm{He}$ white dwarf merger as the best viable model for the majority of the $\mathrm{RCrB}$ stars. On the other hand, the LTP scenario can be ruled out, because it does not result in a helium-rich surface abundance as encountered in KPD 0005+5106 (Werner \& Herwig 2006). Thus it seems inevitable that both KPD $0005+5106$ and the RCrB stars are mergers and form a distinct evolutionary post-AGB sequence. This would be a third sequence with the canonical hydrogen-rich and the LTP-initiated hydrogen-deficient sequences (PG1159 and [WC] stars).

If true, then we expect similar element abundances in KPD 0005+5106 and the RCrB stars. We compare them in Table 5. We used the abundances determined for the so-called majority RCrB stars (Rao \& Lambert 1996), except for neon, which is determined only in a few RCrB stars. We selected the abundance of Y Mus, the only member of the majority RCrBs for which Asplund etal. (2000) could measure the Ne abundance. The uncertainties are in the range $0.2-0.5 \mathrm{dex}$, varying from element to element. There seems to be surprisingly good agreement between the RCrB and KPD 0005+5106 abundances, except for calcium and iron. Calcium, however, has the greatest uncertainty in our analysis (about $1 \mathrm{dex}$ ). More difficult to reconcile are the solar iron abundance in KPD 0005+5106 and the 1 dex subsolar iron abundances in $\mathrm{RCrBs}$.

Acknowledgements. T.R. is supported by the German Aerospace Center (DLR) under grant 05 OR 0806. Some of the data presented in this paper were obtained from the Multimission Archive at the Space Telescope Science Institute (MAST). This work made use of the profile-fitting procedure OWENS developed by M. Lemoine and the FUSE French Team. This research made use of the SIMBAD Astronomical Database, operated at the CDS, Strasbourg, France. This publication makes use of data products from the Two Micron All Sky Survey, which is a joint project of the University of Massachusetts and the Infrared Processing and Analysis Center/California Institute of Technology, funded by the National Aeronautics and Space Administration and the National Science Foundation.

\section{References}

Althaus, L. G., Panei, J. A., Miller Bertolami, M., et al. 2009, ApJ, 704, 1605 Asplund, M., Gustafsson, B., Lambert, D. L., \& Rao, N. K. 2000, A\&A, 353, 287

Asplund, M., Grevesse, N., Sauval, A. J., \& Scott, P. 2009, ARA\&A, 47, 481 
D. Wassermann et al.: Metal abundances in the hottest known DO white dwarf (KPD 0005+5106)

Cardelli, J. A., Clayton, G. C., \& Mathis, J. S. 1989, ApJ, 345, 245

Clayton, G. 1996, PASP, 108, 225

Cohen, M., Megeath, S. T., Hammersley, P. L., Martín-Luis, F., \& Stauffer, J. 2003a, AJ, 125, 2645

Cohen, M., Wheaton, W. A., \& Megeath, S. T. 2003b, AJ, 126, 1090

De Marco, O., Clayton, G., Herwig, F., et al. 2002, AJ, 123, 3387

Dere, K. P., Landi, E., Mason, H. E., Monsignori Fossi, B. C., \& Young, P. R. 1997, A\&AS, 125, 149

Dere, K. P., Landi, E., Young, P. R., et al. 2009, A\&A, 498, 915

Downes, R. A., Liebert, J., \& Margon, B. 1985, ApJ, 290, 321

Feibelman, W. A. 1999, PASP, 111, 22

Feibelman, W. A., \& Bruhweiler, F. C. 1990, ApJ, 357, 548

Fitzpatrick, E. L., \& Massa, D. 2007, ApJ, 663, 320

Fitzpatrick, E. L., \& Massa, D. 2009, ApJ, 699, 1209

Hügelmeyer, S. D., Dreizler, S., Homeier, D., et al. 2006, A\&A, 454, 617

Hummer, D. G., Berrington, K. A., Eissner, W., et al. 1993, A\&A, 279, 298

Jahn, D., Rauch, T., Reiff, E., et al. 2007, A\&A, 462, 281

Kurucz, R. L. 2009, in Recent Directions in Astrophysical Quantitative Spectroscopy and Radiation Hydrodynamics, ed. I. Hubeny, J. M. Stone, K. MacGregor, \& K. Werner, AIP Conf. Proc., 1171, 43
Miksa, S., Deetjen, J. L., Dreizler, S., et al. 2002, A\&A, 389, 953

Rao, N. K., \& Lambert, D. L. 1996, in Hydrogen-Deficient Stars, ed. C. S. Jeffery, \& U. Heber, ASP Conf. Ser., 96, 43

Rauch, T., \& Deetjen, J. L. 2003, in Stellar Atmosphere Modeling, ed. I. Hubeny, D. Mihalas, \& K. Werner, ASP Conf. Ser., 288, 103

Rybicki, G. B., \& Hummer, D. G. 1991, A\&A, 245, 171

Saio, H., \& Jeffery, C. S. 2002, MNRAS, 333, 121

Seaton, M. J., Yan, Y., Mihalas, D., \& Pradhan, A. K. 1994, MNRAS, 266, 805

Skrutskie, M. F., Cutri, R. M., Stiening, R., et al. 2006, AJ, 131, 1163

Unglaub, K., \& Bues, I. 2000, A\&A, 359, 1042

Werner, K., \& Herwig, F. 2006, PASP, 118, 183

Werner, K., Heber, U., \& Fleming, T. 1994, A\&A, 284, 907

Werner, K., Dreizler, S., Heber, U., et al. 1996, A\&A, 307, 860

Werner, K., Dreizler, S., Deetjen, J. L. et al. 2003, in Stellar Atmosphere Modeling, ed. I. Hubeny, D. Mihalas, \& K. Werner, ASP Conf. Ser., 288, 31

Werner, K., Rauch, T., \& Kruk, J. W. 2007, A\&A, 474, 591

Werner, K., Rauch, T., \& Kruk, J. W. 2008, A\&A, 492, L43

Werner, K., Rauch, T., \& Kruk, J. W. 2010, ApJ, 719, L32 